\title{
Qualitative und quantitative Bestimmung der Kohlenhydrate im Urin durch Dünnschichtchromatographie')
}

\author{
Von W. von Berg \\ Aus der Universitäts-Kinderklinik Göttingen (Direktor Prof. Dr. G. Joppich)
}

(Eingegangen am 27. Juni 1968)

\begin{abstract}
Eine dünnschichtchromatographische Methode zur Trennung und grobquantitativen Bestimmung von klinisch wichtigen Kohlenhydraten im Urin wird angegeben. Die Trennung erfolgt auf Cellulose mit Essigester - Pyridin - Wasser, mit nachfolgender kombinierter Anfärbung durch Anilin-Phthalat/Naphthoresorcin-Trichloressigsäure. Die quantitative Auswertung zwischen und nach beiden Färbungen wird durch visuellen Vergleich von Farbintensität und UV-Fluoreszenz der Zucker-Flecken mit gleichzeitig aufgetragenen Testzucker-Gemischen vorgenommen.
\end{abstract}

\section{Qualitative and quantitative determination of urinary carbobydrates by thin layer chromatograpby}

A thin layer chromatographic method is reported for the separation and roughly quantitative determination of clinically important urinary carbohydrates. The separation is performed on cellulose with ethyl acetate - pyridine - water, and the carbohydrates are located by treatment with aniline-phthalate and then with naphthoresorcinol-trichloracetic acid. The quantitative evaluation between and after the two treatments is performed visually by comparing the colour intensity and UV-fluorescence of the sugar spots with those from a mixture of test sugars run simultaneously.

Die ersten Untersuchungen über die Trennung von Kohlenhydraten durch die von STAHL eingeführte Dünnschichtchromatographie erschienen $1961(1,2,3)$. Seitdem sind zahlreiche Arbeiten über dieses Thema veröffentlicht worden (Ubersicht bei 4, 5).

Als Träger werden Kieselgel oder Kieselgur angegeben, sowohl ohne Zusätze zur Trennung von Urinzuckern (6) und Zuckerderivaten (7), als auch imprägniert mit Natrium-Bisulfit (8), Natrium-Phosphat $(9,10,11)$, Natrium-Acetat $(3,12,13,14)$, Natrium-Borat $(15,16)$, bzw. Borsäure $(1,2,13,17,18)$. Auch andere Trägersubstanzen, wie Gips (19) oder Polyamid (20) werden empfohlen.

Anorganische Schichten haben den Vorteil, daß aggressive Substanzen wie Schwefelsäure oder andere mineralsaure Sprühreagenzien zur Sichtbarmachung der Zuckerflecke verwendet werden können. Von Nachteil ist jedoch die z. T. recht unbefriedigende Trennung. Besonderes Gewicht muß aus klinischer Sicht darauf gelegt werden, daß Galaktose und Glucose und auch Fructose scharf voneinander unterscheidbar sein müssen, was durch geeignete Fließmittelkombinationen und Pufferung der Sorptionsschicht auch auf Kieselgel oder Kieselgur durchaus möglich ist.

Eine Trennung dieser drei für klinische Fragestellungen wichtigen Kohlenhydrate kann ferner auf einer Celluloseschicht erreicht werden. Kohlenhydrat-Dünnschichtchromatographien mit diesem Träger wurden 1962 von SCHWEIGer (21) angegeben. Seitdem sind auch damit zahlreiche Untersuchungen durchgeführt worden $(7,22-26)$, sowie mit mikrokristalliner Cellulose (27, 28).

Die Notwendigkeit, schnell und genau einen im Urin ausgeschiedenen Zucker nachweisen und identifizieren zu können, führte uns nach unbefriedigenden Vorversuchen mit gepufferten und ungepufferten Kieselgel G-Schichten zu der von SCHWEIGER (21) angegebenen

1) Teilweise vorgetragen auf dem VI. Internationalen Kongreß für Klinische Chemie, München, 26.-30. Juli 1966.
Kohlenhydrat-Dünnschichtchromatographie mit Cellulose als Trägersubstanz. Entscheidend war dabei insbesondere die gute Trennung von Glucose und Galaktose. Als bedeutungslosen Nachteil muß man jedoch in Kauf nehmen, daß aggressive Sprühreagenzien wegen Verfärbungen der Cellulose nicht verwendet werden können.

\section{Methodik}

DC-Platten: $15 \mathrm{~g}$ Cellulosepulver MN 300 (Macherey, Nagel \& Co., Düren) werden mit 90 bis $100 \mathrm{ml}$ dest. Wasser vermischt und 1 Min. mit einem handelsüblichen Mixgerät homogenisiert. Zur Beschichtung von 5 Platten $(20 \times 20 \mathrm{~cm}$, Schichtdicke $0,25 \mathrm{~mm})$ benutzen wir ein DESAGA-Streichgerät (C. Desaga GmbH, Heidelberg). Nach Lufttrockung über Nacht werden die Platten $10 \mathrm{Min}$. bei $105^{\circ}$ nachgetrocknet und anschließend im Exsikkator aufbewahrt.

Das Fließmittelgemisch besteht aus Essigester / Pyridin / Wasser $\cdot(40: 20: 40 \nabla / v)$, von dem beide Phasen verwendet werden(29). $\mathrm{Zu}$ beachten ist, daß sich das Fließmittelgemisch vor dem Einstellen der Platten $10 \mathrm{Min}$. im Chromatographietrog in seine beiden Phasen trennen muß und nicht wieder aufgeschüttelt werden darf.

Die Auftrennung exfolgt eindimensional, $11 \mathrm{~cm}$ aufsteigend in zwei aufeinanderfolgenden Läufen mit $Z$ wischentrocknung im zimmerwarmen Luftstrom. Jeder Lauf dauert 60 bis $80 \mathrm{Min}$.

Zum Nachweis der Kohlenhydratflecken werden bei uns zwei sich ergänzende Färbungen, die auf derselben Platte vorgenommen werden, durchgeführt:

Zunächst wird mit Anilin-Phthalat (30) besprüht: $995 \mathrm{mg}$ Phthalsäure werden in $60 \mathrm{ml}$ wassergesättigtem n-Butanol gelöst und danach $0,55 \mathrm{ml}$ Anilin zugesetzt. Zur Farbentwicklung werden die besprühten Platten 10 Min. lang auf $105^{\circ}$ (Trockenschrank) erhitzt.

Die auftretenden Farbflecke sowie deren Fluoreszenz im langwelligen UV-Licht (366 nm) (Mineralight, UVSL-13, San Gabriel, Californien) zeigt Tabelle 1 .

Nach Auswertung der Chromatogramme werden die Platten anschließend mit Napthoresorcin-Trichloressigsäure (31) besprüht:

Lösung I: $5 \mathrm{~g}$ Trichloressigsäure, gelöst in $25 \mathrm{ml}$ Wasser,

Lösung II: $50 \mathrm{mg}$ Naphthoresorcin, gelöst in $25 \mathrm{ml}$ absol. unvergälltem Athylalkohol. Vor Gebrauch werden beide Lösungen gemischt und das damit besprühte Chromatogramm zur Farbentwicklung ebenfalls $10 \mathrm{Min}$. auf $105^{\circ}$ erhitzt. 
Tab. 1

Farbqualität, Fluoreszenz und Nachweisempfindlichkeit von Kohlenhydraten bei Färbung mit Anilin-Phthalat allein

\begin{tabular}{|c|c|c|c|c|}
\hline & \multicolumn{2}{|c|}{ Tageslicht } & \multicolumn{2}{|c|}{ UV-Licht (366 nm) } \\
\hline & Farbe & $\begin{array}{l}\text { untere } \\
\text { Nachweis- } \\
\text { Grenze } \\
\mu \mathrm{g}\end{array}$ & Fluoreszenz & $\begin{array}{l}\text { untere } \\
\text { Nachweis- } \\
\text { Grenze } \\
\mu \mathrm{g}\end{array}$ \\
\hline $\begin{array}{l}\text { Lactose } \\
\text { Galaktose } \\
\text { Saccharose } \\
\text { Glucose } \\
\text { Fructose } \\
\text { Arabinose } \\
\text { Xylose } \\
\text { Ribose }\end{array}$ & $\begin{array}{c}\text { braun } \\
\text { braun } \\
\varnothing \\
\text { braun } \\
\text { hellbraun } \\
\text { rotbraun } \\
\text { rotbraun } \\
\text { rotbraun }\end{array}$ & $\begin{array}{l}1,0 \\
0,3 \\
\varnothing \\
0,3 \\
3,0 \\
0,05 \\
0,05 \\
0,05\end{array}$ & $\begin{array}{l}\text { gelbgrün } \\
\text { gelbgrün } \\
\varnothing \\
\text { gelbgrün } \\
\text { schwach gelbgrün } \\
\text { rötlich } \\
\text { rötlich } \\
\text { rötlich }\end{array}$ & $\begin{array}{l}0,5 \\
0,1 \\
\varnothing \\
0,1 \\
3,0 \\
0,1 \\
0,1 \\
0,1\end{array}$ \\
\hline
\end{tabular}

Tab. 2

Farbqualität, Fluoreszenz und Nachweisempfindlichkeit von Kohlenhydraten bei kombinierter Färbung mit Anilin-Phthalat und Naphthoresorcin-Trichloressigsäure

\begin{tabular}{|c|c|c|c|c|}
\hline & \multicolumn{2}{|c|}{ Tageslicht } & \multicolumn{2}{|c|}{ UV-Licht (366 nm) } \\
\hline & Farbe & $\begin{array}{c}\text { untere } \\
\text { Nachweis- } \\
\text { Grenze } \\
\mu \mathrm{g}\end{array}$ & Fluoreszenz & $\begin{array}{l}\text { untere } \\
\text { Nachweis- } \\
\text { Grenze } \\
\mu \mathrm{g}\end{array}$ \\
\hline $\begin{array}{l}\text { Lactose } \\
\text { Galaktose } \\
\text { Saccharose }\end{array}$ & $\begin{array}{c}\text { braun } \\
\text { schokol. braun } \\
\text { blau }\end{array}$ & $\begin{array}{l}1,0 \\
0,3 \\
1,0\end{array}$ & $\begin{array}{l}\text { ziegelrot } \\
\text { ziegelrot } \\
\text { dunkel auf }\end{array}$ & $\begin{array}{l}2,0 \\
0,5 \\
1,0\end{array}$ \\
\hline $\begin{array}{l}\text { Glucose } \\
\text { Fructose } \\
\text { Arabinose } \\
\text { Xylose } \\
\text { Ribose }\end{array}$ & $\begin{array}{c}\text { schokol. braun } \\
\text { rot bis rotbraun } \\
\text { gelbbraun } \\
\text { gelbbraun } \\
\text { gelbbraun }\end{array}$ & $\begin{array}{l}0,3 \\
1,0 \\
0,1 \\
0,1 \\
0,1\end{array}$ & $\begin{array}{c}\text { ziegelrot } \\
\varnothing \\
\varnothing \\
\varnothing \\
\varnothing\end{array}$ & $\begin{array}{c}0,5 \\
\varnothing \\
\varnothing \\
\varnothing \\
\varnothing\end{array}$ \\
\hline
\end{tabular}

Die dann bei den schon bestehenden Flecken beobachteten Farbänderungen sowie die neu auftretenden Flecken sind in Tabelle 2 aufgeführt.

Auf jeder Platte werden neben den Urinproben gleichzeitig je $1 \mu l$ dreier Testzuckergemiscbe steigender Konzentration zum Vergleich aufgetragen, wie BICKEL und SouchoN (32) es analog für die Papierchromatographie von Kohlenhydraten angegeben haben. Das Gemisch besteht aus chromatographisch reiner Lactose, Galaktose, Glucose, Fructose, Xylose und Ribose. Die Konzentrationen in den drei Gemischen betragen je 3, 6 und $9 \mu \mathrm{g}$ Lactose und Fructose/ $\mu l$, bzw. je 1,2 und $3 \mu \mathrm{g} / \mu l$ bei den übrigen Kohlenhydraten. Falls erforderlich werden Arabinose und Saccharose separat aufgetragen.

Vor dem Auftragen der Urinproben wird mit der BENEDIKTschen Probe grob-quantitativ der Gehalt an reduzierenden Substanzen im Urin ermittelt. Ist die Probe negativ, werden $10 \mu l$ entsalzten Urins aufgetragen. Tritt eine grüne oder gelbe Verfärbung auf, werden nur 1 bis $5 \mu l$ Urin verweridet, um eine Úberladung des Chromatogramms zu vermeiden. Bei Braunfärbung ist der Urin vor der Entsalzung 1:10 mit Wasser zu verdünnen. Von dieser Verdünnung werden 1 bis $5 \mu l$ aufgetragen.

Die Entsalzung erfolgt mit einem Mischbett-Ionenaustauscher (Ionenaustauscher $\mathrm{V}, \mathrm{Fa}$. Merck AG, Darmstadt). In einem Zentrifugenglas wird etwa $1 \mathrm{ml}$ Urin zu soviel Ionenaustauscher gegeben, daß der Urin 2 bis $3 \mathrm{~mm}$ übersteht. Dieses Gemisch muß gut durchgeschüttelt werden und bleibt danach etwa 1 Std., aber nicht länger als $12 \mathrm{Stdn}$., stehen. Nach Zentrifugieren kann der klare, farblose Ubberstand unter mäßig warmem Luftstrom auf die Cellulose-Platte aufgetragen werden.

Die Auftragungsstellen der Urinproben und der drei Testzuckergemische sind $3 \mathrm{~cm} \cdot$ vom unteren Rand und untereinander $1,5 \mathrm{~cm}$ entfernt, so daß auf einer Platte bis zu 10 Urinproben gleichzeitig untersucht werden können.

\section{Ergebnisse}

Die $R_{\mathrm{F}}$-Werte nehmen in der Reihenfolge DisaccharidHexose-Pentose zu (Tab. 3).

Dabei lassen sich die Kohlenhydrat-Paare Saccharose/ Galaktose und Fructose/Arabinose nicht trennen. Sie können aber wegen ihrer verschiedenen Farbqualität.
Tab. 3

$R_{\mathrm{F}-W e r t e}$ der Kohlenhydrate auf Cellulose $M N 300 \mathrm{im}$ System Essigester/Pyridin/Wasser (40:20:40 v/v)

\begin{tabular}{ll}
\hline & $R_{\text {F }}$ \\
\hline Lactose & 0,33 \\
Galaktose & 0,44 \\
Saccharose & 0,45 \\
Glucose & 0,48 \\
Fructose & 0,53 \\
Arabinose & 0,53 \\
Xylose & 0,60 \\
Ribose & 0,66 \\
\hline
\end{tabular}

mit Anilin-Phthalat, aufgrund ihrer unterschiedlichen UV-Fluoreszenz und schließlich durch Nachfärbung mit dem Ketose-Reagenz Naphthoresorcin-Trichloressigsäure unterschieden werden (vgl. Tab. 1, 2 und Abb. 1).

Die unteren Nachweisgrenzen sind in den Tabellen 1 und 2 aufgeführt. Aus Tabelle 1 ist ersichtlich, daß die Nachweisempfindlichkeit der Aldohexosen Galaktose und Glucose unter UV-Licht gegenüber Betrachtung bei Tageslicht um das Dreifache und von Lactose um das Doppelte gesteigert wird. Die kombinierte Färbung mit Anilin-Phthalat/Naphthoresorcin-Trichloressigsäure

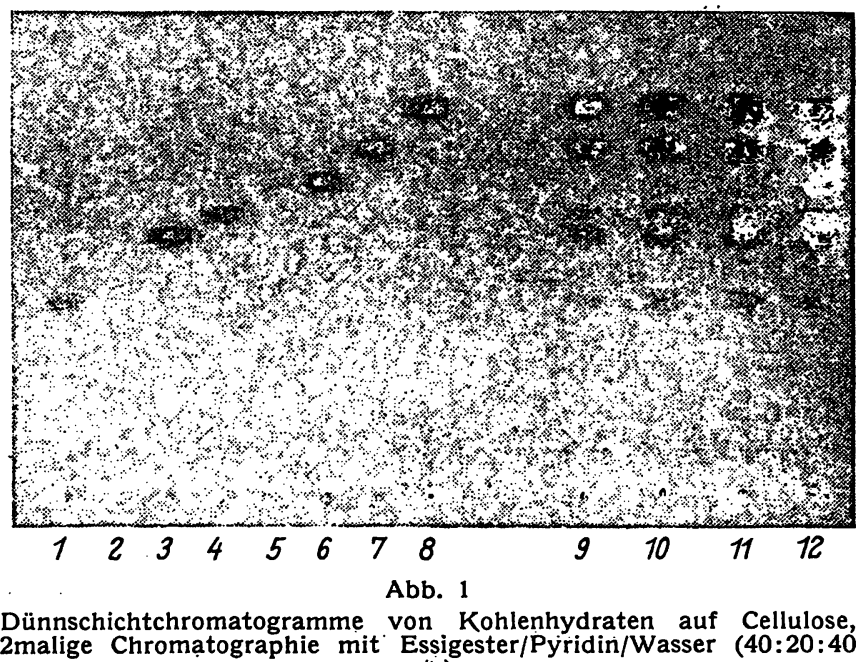

2malige Chromatographie mit Essigester/Pyridin/Wasser (40:20:40 $v / \dot{v})$

a) Anfärbung mit Anilin-Phthalat

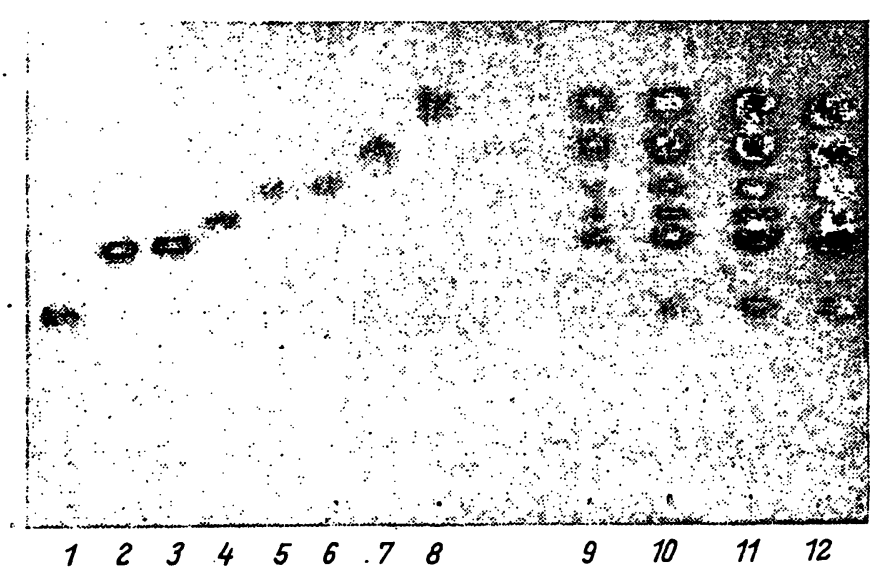

b) Mit Naphthoresorcin-Trichloressigsäure nachgefärbt $1=$ Lactose $(9 \mu \mathrm{g}), 2=$ Saccharose $(10 \mu \mathrm{g}), 3=$ Galaktose $(3 \mu \mathrm{g}), 4=$ Glucose $(3 \mu \mathrm{g}), 5=$ Fructose $(6 \mu \mathrm{g}), .6=$ Arabinose $(1 \mu \mathrm{g}), 7=$ Xylose (1 $\mu \mathrm{g}), 8=$ Ribose $(1 \mu \mathrm{g}), 9=$ Testgemisch von 3, 4, 7, 8 (je (1 $\mu \mathrm{g}$ ) jedoch je $3 \mu \mathrm{g}$ bzw. $9 \mu \mathrm{g}, 12=$ wie 11 , dazu Arabinose $(1 \mu \mathrm{g})$ und jedoch je $3 \mu \mathrm{g}$ bzw. $9 \mu \mathrm{g}, 12=$ wie 11, dazcharose $(10 \mu \mathrm{g})$

Die Auftragsmengen liegen zur besseren Wiedergabe weit über den unteren Nachweisgrenzen 
hat den Vorteil, daß Fructose gut dreimal empfindlicher reagiert als mit Anilin-Phthalat allein. Außerdem wird dadurch die mit Anilin-Phthalat nicht anfärbbare Saccharose deutlich blau sichtbar (Tab. 2 und Abb. 1).

Durch die gleichzeitig mit den Urinproben mitlaufenden Testzuckergemische verschiedener Konzentration sind auch quantitative Angaben über die im Urin ausgeschiedenen Kohlenhydrate möglich. Dazu wird die Farbintensität bzw. die UV-Fluoreszenz der Testzucker mit dem entsprechenden Fleck der Urinprobe visuell verglichen. Für klinische Belange ist diese Methode hinreichend genau, um grobe Abweichungen von der Norm zu erkennen.

\section{Diskussion}

Die angegebene Methode der Kohlenhydrat-Dünnschichtchromatographie am Urin hat sich bei uns in dreijähriger Routine bewährt. Sie ist besonders gut dazu geeignet, bei ungeklärten Melliturien oder bei Verdacht auf eine angeborene Kohlenhydrat-Stoffwechselstörung die Art des ausgeschiedenen Zuckers schnell und einfach zu klären und darüber hinaus auch hinreichend genau quantitative Aussagen zu machen. Die Dringlichkeit einer schnellen Diagnosestellung wird besonders bei solchen angeborenen Erkrankungen des Kohlenhydrat-Stoffwechsels deutlich, bei denen eine frühżeitig einsetzende Behandlung oft von lebenswichtiger Bedeutung ist.

\section{Literatur}

1. Pastuska, G., Z. analyt. Chem. 179, 427 (1961). - 2. Prey, V., H. Berbalk und M. Kausz, Mikrochem. Verein. Mikrochim. Acta (1961) 968. - 3. Stahi, E. und K. Kaltenbach, J. Chromatogr. 5, 351 (1961). - 4. StAhL, E., Dünnschicht-Chromatographie. Ein Laboratoriumshandbuch. 2. Aufl. Springer, BerlinHeidelberg-New York (1967). - 5. Randerath, K., Dünnschicht-Chromatographie. Verlag Chemie, Weinheim/Bergstr. (1965). - 6. Becker, S. und P. MaY, Amer. J. Clin. Path. 49, 436 (1968). - 7. Bancher, E., H. Scherz und K. Kaindi, Mikrochem. Verein. Mikrochim. Acta (1964) 1043. - 8. ADACHI, S., J. Chromatogr. 17, 295 (1965)..-9. Lombard, A., J. Chromatogr. 26, 283 (1967). - 10. Ovodov, Y. S., E. V. Evtushenko, V. E. Vaskovsky, R. G. Ovodova und T. F. Solov' Eva, J. Chromatogr. 26, 111 (1967). - 11. WALDr, D., J. Chromatogr. 18, 417 (1965). - 12. Bancher, E., H. SchÉRZ und K. KaINDL, Mikrochem. Verein. Mikrochim. Acta (1964), 652. - 13. Prfferi, P. G., Analytic. Chem. 37, 925 (1965). - 14. Stefanis, DE, V. A. und J. G. Ponte jr., J. Chromatogr. 34, 116 (1968). - 15. KäSER, H. und G. MAsERA, Schweiz. med. Wschr. 94, 158 (1964). - 16. Martins, P. M. und Y. P. Dick, J. Chromatogr. 32, 188 (1968). -
17. Baron, D. N. und J. Eçonomidis, J. Clin. Path. London 16, 484 (1963). - 18. Lato, M., B. Brunelli, G. Ciuffini und T. Mezzetri, J. Chromatogr. 34, 26 (1968). - 19. Afronso, A., J. Chromatogr. 27, 324 (1967). - 20. Marais, J. P., J. Chromatogr. 27, 321 (1967). - 21. Schweiger, A., J. Chromatogr. 9, 374 (1962). - 22. Grau, R. und A. Schweiger, Zschr. Lebensmittelunters. 119, 210 (1963). - 23. GüNTHER, H. und A. SCHWEIGER, J. Chromatogr. 17, 602 (1965). - 24. Vomнof, D. W., J. Truitr und T. C. TuCker, J. Chromatogr. 21, 335 (1966). 25. VomhoF, D. W. und T. C. TuCKer, J. Chromatogr. 17, 300 (1965). - 26. Lamkin, W. M., D. N. Ward und E. F. Walborg, Analyt. Biochem. 17, 485 (1966). - 27. Wolfrom, M. L., D. L. Patin und R. M. DE Lederkremer, J. Chromätogr. 17, 488 (1965). - 28. WOLFROM, M. L., R. M. DE LEDER KREMER und G. SChWAB, J. Chromatogr. 22, 474 (1966). - 29. Isher wood, F. A. und M. A. Jerimyn, Biochem. J. 48, 515 (1951). - 30. PARTridge, S. M., Nature (London) 164, 443 (1949). - 31. PARTRIDGE, S..M., Biochem. J. 42, 238 (1948). - 32. Bickel, H. und F. Souchon, Arch. Kinderhk. Stuttgart 31. Beiheft (1955).

Dr. W. von Berg

34 Göttingen, Universitäts-Kinderklinik Humboldtallee 38 . 